Finger, I. (1957). J. gen. Microbiol. 16, 350-359

\title{
Immunological Studies of the Immobilization Antigens of Paramecium aurelia variety 2
}

\author{
BY I. FINGER* \\ Zoological Laboratory, University of Pennsylvania, Philadelphia, \\ Pennsylvania, U.S.A.
}

SUMMARY: An immunological study was made of Paramecium aurelia variety 2. The antigens which produce immobilization antibodies were studied and a survey of the antigenic types, or serotypes, manifested by variety 2 stocks carried out. The eight types found were titrated against several sera. Serotype B, when found in different stocks, was distinguished from stock to stock on the basis of reactions to homologous and heterologous sera. Stock-specific $\mathrm{C}$ serotypes were also found and the conditions for maximum stability of the $\mathrm{C}$ serotype occurring in different stocks determined. It has been shown by absorption experiments that: $(a)$ antiserum against a single serotype has at least two antibodies directed against the immobilization antigen(s); (b) if there are two or more determinant groups in a serotype, these groups are on a single molecule. The possible serological bases for cross-reactions of serotypes controlled by the same genetic locus are discussed.

The functions of the nucleus and cytoplasm in heredity and differentiation have been investigated extensively in Paramecium. Studies by Sonneborn \& LeSuer (1948) and by Sonneborn $(1948,1950 a)$ with varieties 4 and 8 of Paramecium aurelia, and by Beale (1952) with variety 1, were concerned specifically with the inheritance of immobilizing antigens. The work reported here deals with immunological studies of similar antigens found in variety 2 .

Cultures of Paramecium aurelia when injected into rabbits elicit antibody formation against paramecia of the injected type. Serum prepared against clones of paramecia pure for a specific antigenic type will, in proper concentrations, immobilize those organisms which have the same immobilizing antigens as the paramecia injected and will not react with other paramecia. When such resistant forms are in turn used to produce antiserum, the new serum will leave unaffected those paramecia which are of the type used for the first injection. Using this procedure, Sonneborn \& LeSuer (1948) demonstrated that a single stock can give rise to several different antigenic types or serotypes which can be told apart on the basis of their reaction to various sera.

As an essential preliminary to immunological and genetic studies of the serotypes a survey of the antigenic types manifested by variety 2 stocks has been made and the conditions for the stability of certain serotypes determined. Absorption experiments have also been performed in an attempt to determine the basis for cross-reactions of serotypes controlled by the same genetic locus found in different stocks. It has been shown that the immobilization determinant groups found in a specific serotype are located on a single molecule.

* Present address : Departments of Neurology and Microbiology, College of Physicians and Surgeons, Columbia University and the Neurological Institute, Presbyterian Hospital, New York, New York, U.S.A. 


\section{METHODS}

Organisms. The following stocks of Paramecium aurelia variety 2, collected in the United States, were used: 1 from Strickersville, New York; 7, Pinehurst, North Carolina; 21, Woodstock, Maryland; 30, Coates Pond, Maryland; 35, Moscow, Indiana; 53, Twin Lakes, Indiana; 72, Pyote, Texas; 83, Millbrook, New Jersey; 85, Blairstown, New Jersey. A single derived stock, d30-2, was also used; these organisms were obtained by crossing stocks 7 and 30 and inducing in the hybrid offspring a nuclear reorganization, autogamy, which ensured homozygosity at all loci. These exautogamous paramecia were capable of manifesting serotype 30-C.

Cultivation. Stocks and all clones derived from them were kept at temperatures from $12^{\circ}$ to $31^{\circ}$, as the experiment required, in a baked lettuce medium or, less frequently in a $\mathbf{0 . 0 7 5} \%$ Cerophyl infusion (Cerophyl tablets or powder obtained from Cerophyl Laboratories, Kansas City, Kansas, U.S.A.) inoculated with Aerobacter cloacae. Cerophyl medium was prepared by dissolving $11 \cdot 3 \mathrm{~g}$. of Cerophyl in 11 . of distilled water, boiling slowly for 3-4 min. and then filtering through cotton. The filtrate was added, with $17 \mathrm{~g} . \mathrm{Na}_{2} \mathrm{HPO}_{4}$, to $15 \mathrm{l}$. distilled water and autoclaved at $15 \mathrm{lb}$./sq.in. for $30 \mathrm{~min}$. Sonneborn's review of methods in the biology of Paramecium should be consulted for details of cultivation and for other methods mentioned below (Sonneborn, 1950b).

Serological methods. Most of the immunological techniques used in these studies were described in detail by Sonneborn $(\mathbf{1 9 5 0 b})$ or are described below in specific sections of the results.

Absorption experiments were carried out with paramecia grown in 21 . flasks or in $15 \mathrm{l}$. of culture fluid in large jugs. Whole organisms, breis, soluble antigens or lyophilized antigens were added to inactivated $\left(56^{\circ}\right.$ for $30 \mathrm{~min}$.) serum and incubated for $90 \mathrm{~min}$. at $27^{\circ}$. The antigen serum mixture was then centrifuged for $2 \mathrm{~min}$. in a clinical centrifuge at $720 \mathrm{~g}$ and the supernatant fluid titrated for immobilization activity. The paramecia were prepared in the four ways mentioned for these experiments as follows:

(1) Whole organisms were filtered through sixteen layers of cheese cloth before being centrifuged for $2 \mathrm{~min}$. in an International electric oil-testing centrifuge at $450 \mathrm{~g}$ or in a small clinical centrifuge until the desired concentration was reached.

(2) Breis were prepared by concentrating the organisms as above, or by using a modified cream separator which was adjusted to allow a rotor speed of $3450 \mathrm{rev}$./ min. (700 g; Dr J. R. Preer, private communication). A Farm Master electric cream separator, 600 pounds capacity (Sears, Roebuck and Co., Philadelphia, Pennsylvania, U.S.A.), was fitted with a pulley of 3 in. diameter to replace the original 5 in. pulley attached to the motor shaft. An endless belt connected this substitute pulley with the spindle of the rotor so that for every turn of this small pulley the rotor revolved about three times. The cream opening in the rotor was completely closed.

The concentrated suspension of paramecia was emptied from the rotor after 15 l. or less had been centrifuged and was placed in a large beaker or flask so as 
to have a high proportion of the $300 \mathrm{ml}$. of concentrate exposed to air. When the desired concentration was reached, following further centrifugation in an oil-testing centrifuge, the paramecia were homogenized in the cold. The brei was either incubated immediately with serum or kept in a deep freezer until needed.

(3) Soluble antigen was extracted simply by centrifuging either a freshly prepared brei or the stored brei in a refrigerated centrifuge at $24,000 \mathrm{~g}$ for $1 \mathrm{hr}$. at $0^{\circ}$ and using the decanted supernatant fluid as antigen.

(4) Lyophilized antigen was prepared by concentrating whole organisms to c. $15 \%$ pure paramecia by volume. The final volume, not exceeding $15 \mathrm{ml}$., was frozen immediately at $-70^{\circ}$ and placed under vacuum in a glass lyophilizing apparatus for 4-10 hr. The lyophilized antigen was stored with a desiccant in a refrigerator.

\section{RESULTS}

\section{Survey of stocks}

A survey of the antigenic types which variety 2 stocks can manifest has been carried out by Dr J. R. Preer (private communication) using the methods of Sonneborn $(1950 \mathrm{~b})$. The general procedure followed was first to obtain antisera by injecting samples of clones into rabbits. Then samples of a number of clones from the same stock as that of the injected paramecia and from other stocks were exposed to these antisera. Paramecia immobilized were classified as having the same serotype as the paramecia against which the sera were originally prepared. Unaffected paramecia were cultivated separately and samples subsequently injected and the new serum collected. This new serum immobilized paramecia which bore the same immobilization antigens as did the injected paramecia.

Eight serotypes were found in variety 2 stocks: A, B, C, D, E, F, G and H. All of these serotypes were tested with sera prepared against serotypes found in varieties 4 and 8 by Sonneborn. Paramecia which were immobilized in one of the specific antisera were classified as being of that specific serotype which was used in the preparation of the antiserum. In this way variety 2 paramecia were designated as serotypes $\mathbf{A}, \mathbf{B}$ and $\mathbf{G}$ because they were immobilized by Sonneborn's specific anti-A, anti-B and anti-G sera, respectively. Dr J. R. Preer discovered that the five other serotypes were unaffected by Sonneborn's antisera. These serotypes were therefore given different letter designations from those serotypes of varieties 4 and 8 . Table 1 lists the serotypes and the titres, the most dilute concentration in which immobilization occurs, against eight sera.

\section{Cross-reactions between serotypes found in different stocks}

The same serotypes found in different stocks generally cannot be told apart even when several homologous and heterologous sera are used for identification. Thus, paramecia of serotype $\mathbf{G}$, a serotype which has been found in every stock of variety 2 which has been tested, have identical titres with a number of anti-G sera. Serotype E of several stocks has shown a similar uniformity of 
reactions. On the other hand, in variety 1 Beale found genetically homologous serotypes, i.e. serotypes whose specificity is controlled by the same genetic locus, which exhibit almost no immunological cross-reactions (Beale, 1952).

Table 1. Titrations of sera prepared against eight serotypes of

Paramecium aurelia variety 2

\begin{tabular}{|c|c|c|c|c|c|c|c|c|c|c|}
\hline \multirow[b]{2}{*}{ Serum } & \multicolumn{2}{|c|}{$\begin{array}{l}\text { Prepared } \\
\text { against }\end{array}$} & \multicolumn{8}{|c|}{ Stock and serotype exposed to sera } \\
\hline & Stock & Type & 53-A & $53 \times 30-B$ & 30-C & 30-D & 53-E & $7 . s-F$ & 30-G & $5-\mathbf{H}$ \\
\hline 10 & 53 & A & 7,9 & $\mathbf{3}, \mathbf{5}$ &,-- &,-- &,-- &,-- &,-- &,-- \\
\hline 12 & $\begin{array}{l}53 \times 30 \\
\text { hybrid }\end{array}$ & $\mathbf{B}$ & 1,3 & 7,9 &,- 2 & $\cdot \cdot$ &,- 3 & 2,5 & 6,8 &,-- \\
\hline 8 & 30 & $\mathrm{C}$ &,- 2 & . & 7,8 &,-- & 1,2 &,-- &,- 4 &,-- \\
\hline 6 & 30 & $\mathbf{D}$ &,-- & . &,-- & 7,8 &,-- &,-- &,-- &,-- \\
\hline 3 & 53 & $\mathrm{E}$ &,- 1 & . . &,-- &,-- & 7,9 &,-- &,- 1 &,-- \\
\hline 11 & $7 . \mathrm{s}$ & $\mathbf{F}$ &,- 1 &,-- & . . & &,-- & 6,8 &,-- &,-- \\
\hline 7 & 30 & G & 3,5 &,-- &,-- &,-- & 2,3 &,-- & 7,10 &,-- \\
\hline 15 & 5 & $\mathbf{H}$ & . . & . &. & . . & . &. & . & 5,7 \\
\hline
\end{tabular}

The three columns on the left refer to sera. The figures in the body of the table represent the lowest concentration of the serum that will immobilize the animals in $2 \mathrm{hr}$. at $27^{\circ}$ (the first figure given), and the lowest concentration that will retard the animals (the second figure). The figures represent concentrations of serum as follows :

\begin{tabular}{|c|c|c|c|c|c|c|c|c|c|c|}
\hline$\frac{\text { Ml. diluent }+ \text { serum }}{\text { ml. serum }}$ & $12 \cdot 5$ & 25 & 50 & 100 & 200 & 400 & 800 & 1600 & 3200 & 6400 \\
\hline Dilution number & $\mathbf{1}$ & 2 & $\mathbf{3}$ & 4 & 5 & 6 & 7 & 8 & 9 & 10 \\
\hline
\end{tabular}

$(-)$ means no reaction; $(\cdot)$ means not tested.

In the variety 2 stocks, $1,30,50,72$ and 83 in which $B$ serotypes are found, four main types of $\mathbf{B}$ can be distinguished on the basis of the similarities and differences of their reactions with two sera. 83-B and 1-B represent one type, 72-B, 50-B and 30-B three more types (Table 2).

Table 2. Titrations of serotypes $B$ and $C$ found in different stocks of

Paramecium aurelia variety 2

\begin{tabular}{|c|c|c|c|c|c|c|c|}
\hline \multirow[b]{2}{*}{ Serum } & \multicolumn{2}{|c|}{ Prepared against } & \multicolumn{5}{|c|}{ Serotypes tested } \\
\hline & Stock & Serotype & $72-\mathrm{B}$ & 30-B & 50-B & $83-B$ & 1-B \\
\hline \multirow{4}{*}{$\begin{array}{l}F \nsubseteq 6 \\
P \nsubseteq 12\end{array}$} & 72 & B & 4,8 &,- 2 & 3,4 &,-- &,$- \mathbf{3}$ \\
\hline & $53 \times 30$ & $\mathbf{B}$ & 5,8 & 7,9 & 3,5 & 3,5 & 3,5 \\
\hline & & & \multicolumn{4}{|c|}{ Serotypes tested } & \\
\hline & & & 30-C & $85-C$ & $7-C$ & 21-C & \\
\hline $\mathbf{F} \sharp 3$ & d30-2 & C & 6,8 & 5,7 & 4,7 & 4,5 & \\
\hline$P \# 9$ & 7 & $\mathbf{C}$ & 5,8 & 4,6 & 6,9 & 2,4 & \\
\hline
\end{tabular}

Serotype $\mathrm{C}$ also differed in various stocks (Table 2 ). Thus, by the combined use of two sera (F $\$ 3$ and $P \sharp 9$ ) it was possible to differentiate 7-C, 21-C and 30-C. The $\mathrm{C}$ in stock 85 appeared to be virtually identical with that in stock 30. 


\section{Stability of serotype $C$}

Serotype $\mathrm{C}$ was found to be rather unstable in stock 30. However, in the derived line d30-2 (a homozygous line containing genes from stock 7 ) it was quite stable. Consequently, the paramecia manifesting the $30-\mathrm{C}$ serotype in all the experiments reported below were taken from d30-2. In these lines 30-C was quite stable at $17^{\circ}, 27^{\circ}$ and $31^{\circ}$, with $17^{\circ}$ the most favourable. Rate of feeding had no effect on stability. The difference in stability of serotype 30-C in stock 30 and line d30-2 suggests that genes from stock 7 play an important part in determining the stability of this serotype (see Sonneborn, Ogasawara \& Balbinder, 1953). Indeed serotype $\mathrm{C}$ is considerably more stable in stock 7 than in stock 30. It is most stable, however, in $\mathrm{d} \mathbf{3 0 - 2}$. In stock 7 it is quite stable at $12^{\circ}$ and $17^{\circ}$ but transforms to another serotype at $27^{\circ}$ and higher. To provide an environment in which both serotypes 30-C and 7-C had approximately equal opportunity for expression, clones were placed at $17^{\circ}$ with enough culture fluid to permit fission rate of $0.5,1 \cdot 0$, or 1.5 fissions/day.

\section{Immunological basis for cross-reactions}

The relationship of immobilizing antigens of the $\mathrm{C}$ serotype to homologous and heterologous sera was studied to determine the basis for cross-reactions of serotypes controlled by a single genetic locus. Two hypotheses will be considered as possible explanations for the observed cross-reactions. One assumes quantitative differences in the antigens, the other assumes qualitative differences. According to either hypothesis, however, a serum prepared against one antigen possesses more than one kind of antibody that will react with this antigen.

A series of absorption experiments was performed to determine whether serum prepared against one serotype has more than one kind of immobilizing antibody against this serotype. When antigens, either in the form of whole organisms, breis, soluble antigen or lyophilized antigen, were added to homologous antiserum and the serum titrated for the ability to immobilize 7-C and 30-C organisms, both heterologous and homologous titres were greatly decreased or completely removed. When the proper concentrations of paramecia of the heterologous serotype were chosen, it was possible to absorb out completely all heterologous activity (five dilutions, i.e. from 1:200 to 0 ) from this same antiserum without lowering the homologous titre more than two dilutions (Fig. 1). If only one type of antibody were present, lowering the titre against one serotype by two dilutions should lower the titre against all cross-reacting serotypes a like amount. Therefore, at least two kinds of immobilizing antibodies must be elicited by the injection of organisms of a single serotype.

Now, according to the first hypothesis, a multiplicity of determinant groups may be present in a serotype, and although all may be present in each serotype, their ratio may vary in the two. If separate antibodies were formed against the various groups, then cross-reactions would be obtained. Absorption of antiserum by a sufficient quantity of heterologous antigen should completely 
remove all antibodies. The first hypothesis, then, requires the demonstration of the presence of the same immobilizing antigens in cross-reacting serotypes. According to this hypothesis large numbers of 7-C paramecia when added to 30-C antiserum should be able to remove completely all immobilization anti-

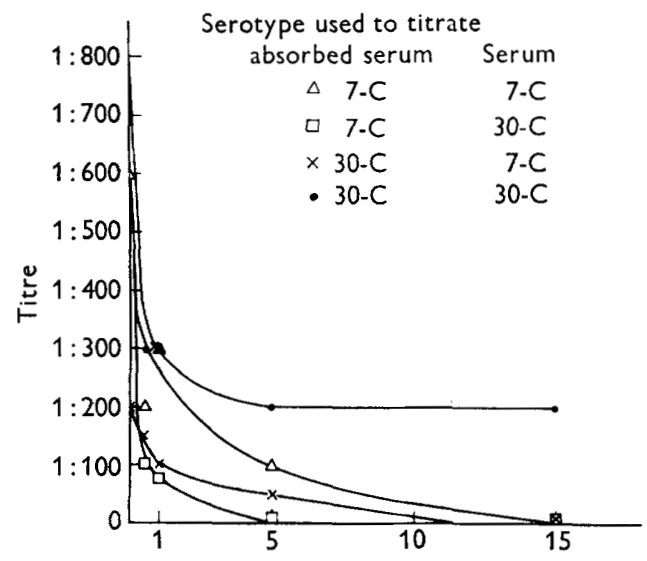

No. of $7-C$ whole paramecia $\left(\times 10^{5}\right)$

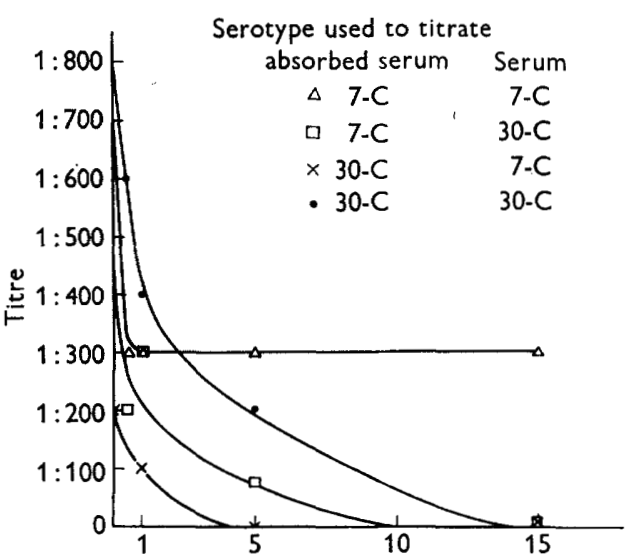

No. of $30-\mathrm{C}$ whole paramecia $\left(\times 10^{5}\right)$

Fig. 1. Absorption of anti- $C$ serum with different kinds of serotype absorbents. The absorbent concentration is given in terms of numbers of paramecia/ml. serum. The curves represent the change in immobilization titre of a serum against a particular $\mathrm{C}$ serotype after serum and absorbent had been incubated. Absorption with 7-C antigen preparations gave comparable curves, including 7-C soluble antigen.

body against 30-C paramecia. But even when 20,000,000 lyophilized paramecia $/ \mathrm{ml}$. serum were added to $30-\mathrm{C}$ antiserum, the quantity of $30-\mathrm{C}$ antibodies was not lowered below dilution 4 or 5 , a level first reached with as few as 50,000 paramecia $/ \mathrm{ml}$. serum. The existence of a plateau which cannot be lowered indicates that there is at least one antibody present in homologous serum with which heterologous organisms do not react. The proportion of all the immobilizing antibodies in the serum that can combine with homologous 
organisms only determines where this plateau levels off for a particular serum. Thus, a serum whose homologous titre can only be decreased from dilution 6 to 4 by a cross-reacting serotype has $25 \%$ of its content antibody unable to react with heterologous organisms (100 (dilution 4):400 (dilution 6)). This $25 \%$ therefore represents that antibody which combines with antigen present in only one of the serotypes, the homologous type. It may, therefore, be concluded that the first hypothesis, requiring the presence of different amounts of the same antigens in cross-reacting serotypes cannot, by itself, account for cross-reactivity.

It appears, then, by elimination of the first hypothesis as likely to account for the cross-reactions of serotypes, that an alternative explanation of a qualitative difference between serotypes is more reasonable. For instance, a multiplicity of determinant groups may be present, some common to both cross-reacting antigens, while others are specifically located in only one. If antibody were formed against common groups, it would react with both antigens. Antibody formed against the specific groups would react with only one of the antigens. That antibodies with such specificities are indeed formed in the case of azo-substituted proteins has been shown by Heidelberger \& Kendall (1934); absorption of antiserum by heterologous antigen should remove only the common antibody leaving behind the specific antibody. Hooker \& Boyd (1934) interpreted their results with hen and duck albumin according to this hypothesis.

\section{Location of immobilization determinant groups}

If at least two immobilization combining groups are responsible for the specificity of a serotype, one cross-reactive group and one group not reacting with other serotypes controlled by the same locus, it would be of interest to know whether these combining groups for specific reactions and for crossreactions are on a single molecule. To determine the locations of these groups the following experiment was performed.

A soluble antigen was extracted from 7-C paramecia and added to 30-C antiserum so that almost all 7-C antibodies would be removed while, at the same time, the 30-C titre would remain high. To the absorbed supernatant fluid was added 7-C antiserum which had previously been absorbed with enough extract of 30-C paramecia to remove almost all the 30-C antibodies, as determined by immobilization tests, and to leave almost unaltered the 7-C titre. The antigen concentration for both $7-\mathrm{C}$ and $30-\mathrm{C}$ paramecia was 200,000 paramecia/ml. undiluted serum. After incubation the mixed sera were centrifuged and the supernatant fluid titrated with the results shown in Table 3.

If the immobilization antigens had been on separate molecules, the 30-C antiserum absorbed with 7-C antigen should have in its supernatant 7-C specific antigen since this antigen would not be brought down with the fraction that would remove the cross-reacting antibodies. Similarly, the absorbed 7-C antiserum would also contain $30-\mathrm{C}$ specific antigen. When the two absorbed sera are mixed the titre of this mixed serum against both 7-C and 30-C paramecia should be lowered. Instead, the serum behaved as though its two component 
sera had no antigen molecules present: the titre of the mixed absorbed sera remained high against both serotypes. The alternative expectations for this experiment are shown in Fig. 2. Apparently, then, if there are two immobilization combining groups in a serotype, they behave as a unit. When one combining group is bound to an antibody, as in absorption, the other immobilization-combining groups are also tied up even though not reacting with the antibody.

Table 3. Titration of supernatant mixtures of absorbed antisera for presence of free antibody

The titres are expressed as the reciprocals of the dilutions of the sera.

$\begin{array}{cc}\text { Titre against } & \text { Titre against } \\ \text { 30-C paramecia } & 7-C \text { paramecia } \\ 200-400 & 50-100 \\ 25-50 & 100-200 \\ 50-100 & 12 \cdot 5 \\ 12 \cdot 5 & 50 \\ 100-200 & 50-100\end{array}$

Unabsorbed 30-C antiserum

Unabsorbed $7-\mathrm{C}$ antiserum

30-C antiserum absorbed with 7-C paramecia

7-C antiserum absorbed with $30-\mathrm{C}$ paramecia

Mixture of the supernatant fluids of the two

absorbed sera

Two reacting groups on a single molecule

Two reacting groups on different molecules
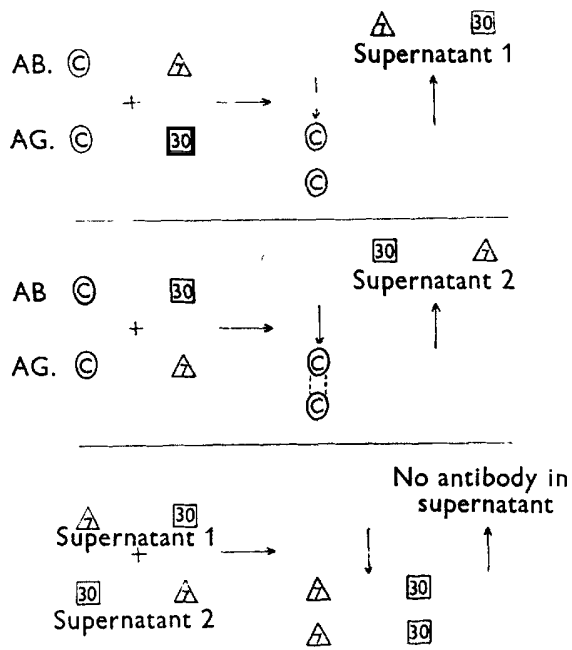

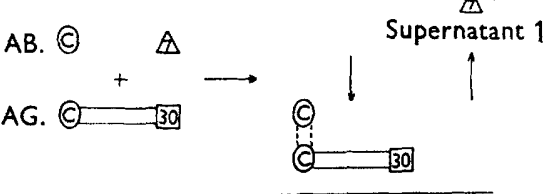

30
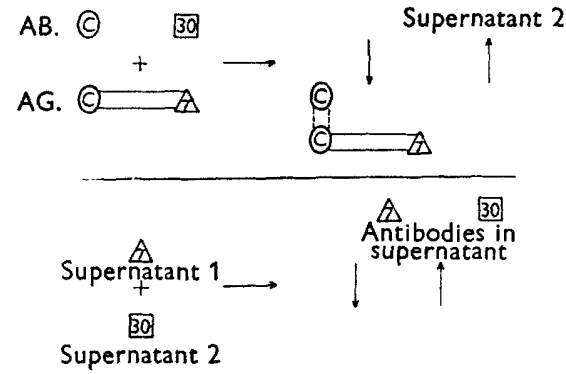

Fig. 2. Absorption experiment to determine if determinant groups are on separate molecules. $\mathbf{A B} .=$ antibody. $\mathbf{A G} .=$ antigen.

\section{DISCUSSION}

Although only one qualitative difference between antigens has been suggested above (that involving common and specific determinant groups as explaining cross-reactions) there is another possible qualitative mechanism which does not assume separate antibodies against distinct determinant groups. The two antigens might be closely related but show small chemical or structural differences. Antibodies would be directed against the antigen molecule as a 
whole, but the various antibody molecules would not be homogeneous, differing in their affinity for the homologous antigen. A mechanism such as this was suggested by Landsteiner \& van der Scheer (1936) as the most reasonable explanation for some of their results from using artificial conjugated azoproteins. The basis for cross-reactivity according to this view would reside in the lack of uniform reactivity of the antibody molecules formed against the homologous antigen, for the heterologous and homologous antigens would not be expected to show the same affinities for the different antibody molecules.

The basis for cross-reactivity of a serotype with sera prepared against other serotypes within the same stock (as contrasted with cross-reactions between the same serotype in different stocks) is still unknown. An analysis similar to the one carried out on the stock-specific $\mathrm{C}$ serotypes using absorption techniques would be expected to be of similar value in discovering the cause of this kind of cross-reactivity. However, even if an antigen common to serotypes from different stocks were the cause of the cross-reactions shown by these stock-specific serotypes, it would seem less likely that a common antigen would account for the cross-reactions of serotypes determined by different loci. Alleles at a locus might be expected to control the synthesis of similar products, but this would appear to be less likely in the case of genes situated at entirely different loci.

Sonneborn (1947), on the basis of early absorption studies, suggested that there exist in paramecia of a particular serotype, two classes of antigens. One of these classes, the secondary antigens, does not take part in the immobilization reaction, but can induce the formation of antibodies capable of immobilizing a second serotype. This second serotype possesses this secondary antigen as a primary antigen, one capable of reacting with immobilization antibodies. Such secondary antigens should be capable of detection by absorption experiments. Later more extensive studies carried out with sera possessing high titres of immobilizing antibodies completely failed to reveal secondary antigens, e.g. by comparing absorption with whole paramecia and with breis (Dr T. M. Sonneborn, personal communication).

Experiments reported elsewhere with variety 2 (Finger, to be published), 4 (Sonneborn, 1950 $a$ ) and 1 (Beale, 1952), have demonstrated that serologically related serotypes found in different stocks are determined by alleles. It is not known whether the converse is true, that unrelated serotypes are determined by allelic genes in variety 2 , because the necessary crosses have not been made. Beale's studies with variety 1 (1952) included crosses between both serologically related and serologically unrelated serotypes, and in both instances he found that a single locus might control cross-reactive serotypes and noncross-reactive serotypes. In other words, it is possible to have genetic homology without a corresponding serological homology.

A plausible explanation for the fact that a locus may control serotypes which have almost no serological relationship, as determined by the immobilization test, is that this test may be extremely sensitive. Thus, although serotypes which may have almost identical antigenic configurations cannot be told apart, as may be the case with the $\mathbf{G}$ serotypes, serotypes which differ only slightly. 
such as perhaps the various $\mathrm{B}$ and $\mathrm{C}$ serotypes, may be distinguished with little difficulty. On the other hand, should two serotypes resemble each other only slightly, on the basis of their immobilization reactions they may be classed as distinctly unrelated types.

It may be concluded that a further study of the immunological relationships of cross-reacting serotypes may still be considered a fruitful approach to the problem of gene action. Similarly, the general problem of the differentiation of cells with like genomes may be approached through an immunological investigation of the relation of serotypes controlled by different genetic loci. Although this relationship may not be demonstrated by means of the immobilization test, it is possible that the application of other immunological techniques, such as gel diffusion methods (Preer, to be published; Finger, to be published), may prove valuable in detecting such relationships, if they do indeed exist.

These studies were supported in part by a research grant of the Phi Beta Psi Sorority administered by Dr J. R. Preer. During much of the work the author was a National Science Foundation Predoctoral Fellow and a Philadelphia Brewers Association Fellow.

\section{REFERENCES}

Besle, G. H. (1952). Antigenic variation in P. aurelia, variety 1. Genetics, 37, 62. Heidelberger, M. \& Kendall, F. E. (1934). Quantitative studies on the precipitin reaction. The role of multiple reactive groups in antigen-antibody union as illustrated by an instance of cross-precipitation. J. exp. Med. 59, 519.

Hooker, S. B. \& Boyd, W. C. (1934). The existence of antigenic determinants of diverse specificity in a single protein. II. In two natural proteins; crystalline duck egg albumin and crystalline hen albumin. J. Immunol. 26, 469.

LaNDSTEINER, K. \& VAN Der Scheer, J. (1936). On cross reactions of immune sera to azoproteins. J. exp. Med. 63, 325.

Sonneborn, T. M. (1947). Developmental mechanisms in Paramecium. Growth Symp. 11, 291.

Sonnebonn, T. M. (1948). The determination of hereditary antigenic differences in genically identical Paramecium cells. Proc. nat. Acad. Sci., Wash. 34, 413.

Sonneborn, T. M. (1950a). The cytoplasm in heredity. Heredity, 4, 11.

Sonneborn, T. M. (1950 b). Methods in the general biology and genetics of $P$. aurelia. J. exp. Zool. 113, 87.

Sonneborn, T. M. \& LeSuer, A. (1948). Antigenic characters in P. aurelia (variety 4): determination, inheritance and induced mutations. Amer. Nat. 82, 69.

Sonneborn, T. M., Ogasamara, F. \& Balbinder, E. (1953). The temperature sequence of the antigenic types in variety 4 of Paramecium aurelia in relation to the stability and transformations of antigenic types. Microbiol. gen. Bull. 7, 27. 